\title{
Synthèse
}

\section{Lutte contre les maladies bactériennes de la pomme de terre dues aux Pectobacterium spp. (Erwinia carotovora)}

\author{
Xavier Latour ${ }^{1}$ \\ Denis Faure ${ }^{2}$ \\ Stéphanie Diallo ${ }^{1}$ \\ Amélie Cirou ${ }^{2}$ \\ Bruno Smadja ${ }^{1}$ \\ Yves Dessaux ${ }^{2}$ \\ Nicole Orange ${ }^{1}$
}

${ }^{1}$ Laboratoire de microbiologie du froid "

Signaux et micro-environnement",

UPRES-EA 4312,

Institut universitaire technologique d'Evreux, 55, rue Saint-Germain,

27000 Evreux

France

<xavier.latour@univ-rouen.fr>

$<$ stephanie.diallo@etu.univ-rouen.fr>

<bruno.smadja@univ-rouen.fr>

$<$ nicole.orange@univ-rouen.fr>

${ }^{2}$ Centre national de la recherche scientifique, Institut des sciences du végétal,

UPR2355,

Avenue de la Terrasse,

91198 Gif-sur-Yvette,

France

$<$ denis.faure@isv.cnrs-gif.fr>

$<$ amelie.cirou@isv.cnrs-gif.fr>

$<$ yves.dessaux@isv.cnrs-gif.fr>

\begin{abstract}
Résumé
Les bactéries macergènes appartenant aux espèces Pectobacterium atrosepticum et Pectobacterium carotovorum sont responsables de dégâts importants lors de la culture et de la conservation après récolte des tubercules de pomme de terre. L'espèce bactérienne impliquée et la gravité des pertes dépendent de facteurs environnementaux, en particulier de l'humidité et de la température, ainsi que du potentiel infectieux des sols. Aucun traitement ne permet aujourd'hui de lutter efficacement contre cette maladie. Dans cette revue, nous présentons les travaux récents concernant les méthodes de lutte chimique, génétique et biologique mises en œuvre pour limiter l'action néfaste de ces bactéries. De nouvelles stratégies dont le but est de perturber la communication quorum-sensing des Pectobacteria sont discutées. L'utilisation d'inocula bactériens et/ou de biostimulateurs des populations bactériennes non pathogènes est notamment envisagée pour dégrader les signaux de quorum-sensing produits par l'agent pathogène.
\end{abstract}

Mots clés : bactérie pathogène ; lutte biologique ; Solanum tuberosum.

Thèmes : amélioration génétique ; pathologie ; productions végétales.

\section{Abstract \\ Control of bacterial diseases of potato caused by Pectobacterium spp. (Erwinia carotovora)}

Pectinolytic bacteria belonging to Pectobacterium atrosepticum and Pectobacterium carotovorum species are the causal agents responsible for important damage during the culture and storage of potato tubers. The bacterial species involved and the severity of damages depend on several environmental factors, such as humidity and temperature, as well as infectious potential of soils. There is currently no treatment to effectively control the virulence of the pathogens. In this review, recent works on chemical, genetic and biological strategies developed to limit the harmful action of these bacteria are presented. Novel strategies targeting the quorum-sensing communication of Pectobacteria are also discussed. The use of bacterial inocula and/or bioactivators stimulating the nonpathogenic bacteria are proposed to degrade the quorum-sensing signals of the pathogen.

Keywords: biological control; pathogenic bacteria; Solanum tuberosum.

Subjects: genetic improvement; pathology; vegetal productions.

es cultures de pomme de terre (Solanum tuberosum) et les tubercules récoltés sont sujets à de nombreuses agressions bactériennes. Il s'agit de bactéries appartenant notamment aux genres Clavibacter, Pectobacterium, Ralstonia, et Streptomyces. Parmi ces derniers, un regain d'intérêt est accordé aux bactéries macergènes appartenant au genre Pectobacterium, plus connues sous le nom d'Erwinia carotovora. Ce changement d'appellation est le résultat de l'adoption par la communauté scientifique d'une nouvelle nomenclature basée 
sur les travaux de taxonomistes français en particulier (Gardan et al., 2003) lors de la $\mathrm{XI}^{\mathrm{e}}$ Conférence internationale sur les bactéries pathogènes des plantes (10-14 juillet 2006, Edimbourg).

Les bactéries Pectobacterium atrosepticum et Pectobacterium carotovorum (anciennement $E$. carotovora subsp. atroseptica et E. carotovora subsp. carotovora) sont des pathogènes de qualité qui provoquent des pertes au champ et lors de la conservation des tubercules (pourritures molles). En végétation, ces bactéries sont responsables d'attaques précoces du tubercule mère et des germes empêchant la levée, ou d'attaques plus tardives comme la maladie de la jambe noire (Priou et Jouan, 1996). Outre les baisses de rendement des cultures, les tubercules récoltés atteints de pourriture molle peuvent engendrer des pertes importantes lors du stockage par une contamination rapide des tubercules avoisinants. Enfin, les tubercules jouant aussi le rôle de semence, des tubercules contaminés, même sans symptôme visible (porteurs sains), peuvent disséminer la maladie au champ lors des phases de multiplication des plants. Ces maladies affectent donc toutes les étapes de la filière pomme de terre : la production et la certification des plants, comme la production de pomme de terre de consommation ou de transformation alimentaire ou industrielle.

Les pertes économiques causées par la recrudescence de ces maladies bactériennes et l'absence de moyens de lutte efficaces motivent aujourd'hui le développement de recherches multidisciplinaires visant à protéger du mieux possible les cultures. Dans cette revue, nous faisons le point sur les principales fonctions bactériennes impliquées dans la virulence et sur différentes stratégies de lutte chimique, génétique et biologique disponibles ou en cours d'étude.

\section{Principaux facteurs environnementaux et bactériens de la virulence}

L'apparition et la gravité des dégâts liés à Pectobacterium sont multifactorielles. Elles dépendent de la réceptivité de la culture à la maladie, du potentiel infectieux environnemental et des conditions pédoclimatiques lors de la culture. Ces dernières expliquent, en grande partie, la prédominance de l'une ou l'autre des deux espèces impliquées dans la maladie. En effet, si les symptômes sont toujours favorisés par une atmosphère confinée et humide, par un excès de pluies et par les blessures qui favorisent la pénétration des bactéries, la présence des bactéries $P$. atrosepticum se limite habituellement aux tubercules cultivés dans les sols tempérés, tandis que les bactéries de l'espèce $P$. carotovorum sont très représentées dans les zones plus chaudes, notamment subtropicales et tropicales. Dans le nord et l'ouest de l'Europe, la température la plus favorable à la maladie est ainsi comprise entre 15 et $20^{\circ} \mathrm{C}$ pour $P$. atrosepticum et aux environs de $25^{\circ} \mathrm{C}$ pour $P$. carotovorum. Cela s'explique par une différence d'activité métabolique des espèces impliquées: pour chacune d'elles, c'est dans cette gamme de températures particulières que la capacité de multiplication et de production d'enzymes lytiques est suffisamment élevée, voire optimale, pour causer des dégâts aux cultures (Smadja et al., 2004b).

Lorsque les conditions climatiques sont favorables, les Pectobacteria présents dans le sol et à la surface des végétaux sensibles passent d'un état de latence à un état d'activité favorisé par la blessure des plantes et le relargage de sucs végétaux. La pénétration dans l'hôte est facilitée par ces blessures, mais elle peut aussi se faire du sol vers le tubercule par des ouvertures naturelles comme les lenticelles (Pérombelon, 2002). Dans un premier temps, les populations bactériennes vont d'abord se multiplier. Puis, les bactéries sécrètent, de façon synchrone et en réponse à un mécanisme de régulation détaillé ultérieurement dans cet article, l'ensemble des enzymes lytiques dont l'activité est responsable des symptômes de macération observables sur plants et tubercules. Chez les Pectobacteria, cet arsenal enzymatique est particulièrement bien adapté à la dégradation de tous les composants des parois cellulaires végétales, ce qui explique l'ampleur des dégâts observés et leur vitesse d'apparition. Il s'agit de protéases, de cellulases et de nombreuses enzymes pectinolytiques (pectine et pectate lyases, pectate hydrolases et pectine méthylestérases) (Smadja et al., 2004a).

\section{Lutte chimique}

À ce jour, il n'existe pas de méthode de lutte efficace contre P. atrosepticum et
P. carotovorum. Seules des mesures prophylactiques, basées sur une hygiène générale des exploitations et des semences ainsi que l'utilisation de pratiques culturales raisonnées et peu mécanisées, permettent de limiter les dégâts enregistrés (Priou et Jouan, 1996). En tenant compte des pratiques agronomiques actuelles, notamment des cultures utilisées lors de la rotation et d'études épidémiologiques réalisées récemment au champ, il est possible de limiter les pertes sur pieds dues à ces pathogènes (Hélias, 2008).

Des mesures visant à limiter la présence des pathogènes sur le tubercule après récolte et leur dissémination dans l'environnement sont à l'étude. Par exemple, l'utilisation de réacteurs de décontamination dont la technologie est basée sur l'émission de plasmas froids est envisagée pour traiter les eaux de lavage des tubercules après récolte (Moreau et al., 2005). Au champ, les traitements chimiques des cultures à base de cuivre ou d'organomercure demeurent peu efficaces et sont déconseillés pour des raisons de protection de l'environnement (Priou et Jouan, 1996). De nouveaux composés capables d'altérer l'intégrité cellulaire bactérienne ou d'inhiber la croissance des Pectobacteria ont montré, au contraire, une réelle efficacité lors d'essais au laboratoire: il s'agit de solutions salées de chlorure d'aluminium et de métabisulfite de sodium (Yaganza et al., 2004) ou de peptides de synthèses (Kamysz et al., 2005). Si ces composés peuvent sembler très intéressants grâce à leur large spectre d'hôte (fongique et bactérien), le risque écologique d'altération des équilibres microbiens apparaît très important. La destruction des communautés bactériennes épiphytes (présentes sur les parties aériennes) ou rhizosphériques (associées aux racines) pourrait ainsi entraîner des baisses de rendement par destruction des bactéries bénéfiques et/ou laisser le champ libre au développement de pathogènes encore plus redoutés. À notre connaissance, l'intérêt des molécules décrites dans ces articles n'a pas encore été analysé au champ.

Il est important de souligner que les politiques agricoles de nombreux pays mettent aujourd'hui l'accent sur l'évaluation de l'efficacité des traitements versus leur coût environnemental (Epstein et Bassein, 2003). Pour répondre à cette demande, de nouvelles molécules à faible impact environnemental sont actuellement recherchées, notamment des 
molécules ciblant la virulence du pathogène (antivirulents) plutôt que sa viabilité (bactéricides). Certains aspects de ces travaux sont présentés dans les paragraphes suivants.

\section{Lutte génétique}

L'organisation génétique de la résistance de la pomme de terre aux maladies a été étudiée en détail par Gebhardt et Valkonen (2001): les 19 gènes de résistance dominants, identifiés grâce à des marqueurs $\mathrm{ADN}$, ont trait à la résistance aux virus, nématodes et champignons. Les quelques régions susceptibles de jouer un rôle dans la résistance à $P$. atrosepticum semblent éparpillées sur les 12 chromosomes de cette plante. Ces résultats peuvent, en partie, expliquer pourquoi il n'existe que peu d'espèces du genre Solanum présentant des caractères de résistances aux Pectobacterium spp. et que les niveaux de résistance enregistrés restent peu élevés. L'amplitude de cette résistance ténue varie avec la variété étudiée. Pasco (2005a, 2005b) montre ainsi que sur un panel de 16 variétés, la variété Kerpondy est peu sensible, tandis que la variété Ackergesegen est très sensible aux pourritures molles engendrées par $P$. atrosepticum. Chez la pomme de terre, les mécanismes naturels de résistance contre ces bactéries affectent la croissance et l'invasion bactérienne à l'intérieur du végétal ou limitent la synthèse des enzymes impliquées dans leur pouvoir pathogène (Lyon, 1989). Ces mécanismes sont cependant peu efficaces, en particulier, parce qu'ils requièrent des conditions aérobies pour s'exprimer. Or, les précipitations ou une irrigation forte, caractéristiques de certaines zones de production, peuvent limiter la diffusion de l'oxygène et créer des conditions anaérobies à la fois défavorables à ces mécanismes de défense des plantes et favorables aux métabolismes des pathogènes bactériens (Pérombelon, 2002).

Les connaissances actuelles sur la génétique de résistance de la pomme de terre aux bactéries macergènes sont encore balbutiantes. De nouvelles données sont attendues grâce au séquençage de son génome. Par ailleurs, les contraintes liées à la sélection d'autres caractéristiques variétales sont fortes. En effet, la recherche de traits de résistance ne peut se faire au détriment de celle de caractères améliorant le rendement, la conservation, les critères organoleptiques ou la teneur en fécule ou sucres réducteurs. Ainsi, l'obtention par croisement variétal de plants présentant l'ensemble de ces critères est actuellement difficile, car longue et aléatoire.

Ce constat a amené certains généticiens à proposer d'autres voies de sélection. L'une d'elles passe par la création de variétés transgéniques, c'est-à-dire de pomme de terre ayant intégré un ou plusieurs gènes issus d'une autre espèce microbienne, végétale ou animale. L'une des premières constructions réalisées a consisté à intégrer dans le génome de la pomme de terre un gène originaire d'un bactériophage codant pour la production de lysozyme qui dégrade la membrane de certaines bactéries. La plante modifiée fabrique du lysozyme qui est transporté vers les espaces intercellulaires des tissus végétaux. Cette opération diminue significativement la sensibilité de la variété étudiée aux attaques de $P$. atrosepticum, que ce soit lors d'essais sur tubercules ou sur plantules cultivées en serres. Ces effets bénéfiques sont attribués à la production du lysozyme qui inhiberait la multiplication des bactéries et maintiendrait leur densité en dessous du niveau de virulence (Düring et al., 1993). L'action du lysozyme n'étant pas spécifique de la paroi bactérienne de l'espèce $P$. atrosepticum, il existe un risque environnemental similaire à celui évoqué précédemment pour d'autres molécules antibactériennes à large spectre d'hôtes. Ce risque a été évalué en comparant les communautés microbiennes associées aux variétés Désirée et Grata à celles de leurs lignées transgéniques (Heuer et Smalla, 1999 ; Lottmann et al., 2000 et 1999). Les résultats de ces études suggèrent que les plantes transgéniques ont un faible impact sur la structure des communautés microbiennes, quelles soient épiphytes ou rhizosphériques (racines ou tubercules). Pour les auteurs, les variations liées à la présence du transgène sont mineures au regard des variations observées chez des plantes non transgéniques et dues à l'âge de la plante, la température, l'humidité ou l'action de pesticides. Ces travaux stipulent également que les populations de la phyllosphère sont plus diverses que celles de la rhizosphère et qu'elles constituent donc d'excellents marqueurs biologiques permettant d'évaluer l'impact de transgènes sur les communautés bactériennes. Ainsi, les faibles variations enregistrées au niveau du feuillage constitueraient un argument supplémentaire en faveur du faible impact des lignées transgéniques sur la structure des communautés bactériennes (Heuer et Smalla, 1999). Il reste bien sûr à évaluer les risques de dissémination du transgène lui-même à d'autres plantes ou organismes en contact avec les plantes transgéniques. D'autres propositions d'amélioration des plantes par transgenèse sont discutées plus loin. Ces arguments scientifiques ne doivent cependant pas faire oublier la faible acceptabilité des végétaux génétiquement modifiés en Europe liée à l'existence de controverses scientifiques, de considérations philosophiques prévalentes chez les populations européennes en particulier sur l'acceptabilité des "risques " et à une politique de communication forte des opposants à l'emploi de ces techniques (Wisniewski et al., 2002 ; Forbes, 2006 ; Myskja, 2006 ; Varzakas et al., 2007). Néanmoins, la culture de pommes de terre génétiquement modifiées à des fins de production de molécules d'intérêts pharmaceutiques ou médicales devrait pouvoir avoir une meilleure acceptabilité par la société en répondant à des problèmes de santé humaine (Y. Bègue, directeur du Comité Nord - organisme membre de la Fédération des producteurs de plants de pomme de terre, communication personnelle).

\section{Lutte biologique}

Dès le début des années 1980, Kloepper (1983) proposa d'utiliser les capacités antagonistes de certaines bactéries vis-àvis des Pectobacteria pour protéger les tubercules et les plants de pomme de terre. Les bactéries protectrices ciblées étaient qualifiées de rhizobactéries favorisant la croissance des plantes et appartenaient au groupe des Pseudomonas spp. fluorescents. Les espèces bactériennes de ce groupe se caractérisent par un métabolisme versatile et par une capacité élevée à extraire le fer du sol pour leurs besoins nutritionnels, grâce à des sidérophores particulièrement affines. Ces caractéristiques expliquent en grande partie leur aptitude à s'installer en nombre et durablement sur et à proximité des racines (Latour et Lemanceau, 1997). L'isolement massif de Pseudomonas a permis de sélectionner quelques rares souches de Pseudomonas fluorescens et Pseudomonas putida antagonistes des Pectobacteria in vitro, c'est-à-dire lors d'essais 
d'inhibition sur boîtes de pétri. Ces isolats limitaient aussi l'apparition de pourritures molles sur tubercules (Xu et Gross, 1986 ; Rhodes et Logan, 1987). Le pouvoir protecteur de ces souches était attribué à la production des sidérophores (Xu et Gross, 1986) ou à celle d'antibiotique, comme le 2,4-diacétylphloroglucinol (Cronin et al., 1997). Malheureusement, l'efficacité de ces isolats n'a jamais été démontrée au champ. Ce type d'échec est communément attribué aux différences de caractéristiques biotiques et abiotiques des sols, qui ne permettent pas à ces souches de maintenir dans le temps une population suffisamment élevée pour être efficace (Duffy et al., 2003; Compant et al., 2005). En effet, même si les Pseudomonas antagonistes introduits présentent une capacité élevée à coloniser la plante et le sol, il demeure que ces souches entrent en compétition avec les autres bactéries autochtones primo-installées et généralement mieux adaptées qu'elles aux conditions du milieu, y compris à la lignée végétale à protéger (Latour et al., 2003). Malgré ces échecs lors des essais en champ, cette stratégie ne doit pas être rejetée en bloc. Des applications ciblées sont envisageables, notamment lors de cultures hors sol au cours desquelles les bactéries Pseudomonas représentent environ $10 \%$ de la communauté bactérienne rhizosphérique. Par ailleurs, cette technique d'apport d'agent biologique antagoniste peut être couplée à l'apport d'intrants chimiques spécifiques ou l'utilisation de plantes transgéniques dans des propositions de lutte intégrée. Par analogie avec les techniques d'enrobage de semences, l'apport de ces agents pourrait aussi se faire par aspersion des tubercules mères juste avant leur installation au champ.

\section{Signalisation quorum- sensing et nouvelles perspectives de lutte}

L'expression des facteurs de virulence des Pectobacteria est régulée par un mécanisme original découvert au début des années 1990: le quorum-sensing (Fuqua et al., 1994). Celui-ci permet l'expression des gènes de virulence seulement lorsqu'un quorum bactérien est atteint. À quelle étape du cycle de virulence intervient cette régulation quorumsensing? Après leur pénétration dans les tissus de la plante hôte, généralement par des ouvertures naturelles comme les lenticelles ou par des blessures (Pérombelon, 2002), les bactéries se multiplient jusqu'à atteindre un nombre nécessaire et suffisant. C'est seulement lorsque ce quorum bactérien est atteint, que les bactéries sécrètent, de façon synchronisée, l'ensemble des facteurs de virulence impliqués dans l'altération des composants des parois végétales (Maë et al., 2001).

Afin d'évaluer leur densité et donc de percevoir ce quorum, les Pectobacteria synthétisent des molécules signal. En effet, chaque cellule bactérienne produit des $N$-acylhomosérine lactones (HSL) qui diffusent librement à travers les membranes cellulaires. Lorsque la multiplication bactérienne atteint le quorum, la concentration en signaux HSL dans le milieu devient suffisamment élevée pour être perçue par les bactéries comme un signal d'activation de la synthèse des facteurs de virulence (revues récentes par Waters et Bassler, 2005; Reading et Sperandio, 2006). L'existence du signal de quorumsensing chez toutes les espèces de Pectobacterium macergènes et son importance stratégique dans la maladie (Smadja et al., 2004a) désignent ce signal bactérien comme une cible privilégiée pour développer des outils de lutte contre la virulence des Pectobacteria. Ces nouvelles méthodes de lutte n'ont pas vocation à éliminer ce pathogène, mais à en atténuer la virulence : ce principe est donc appelé antivirulence.

Ces stratégies d'antivirulence ont toutes pour vocation l'interruption de la signalisation quorum-sensing de Pectobacterium: on parle de quorum-quenching. Il s'agit de procédés multiples, parfois complémentaires :

- des composés chimiques qui perturbent la perception par les pathogènes du signal quorum-sensing;

- des agents de biocontrôle sélectionnés pour leur capacité à dégrader les signaux du quorum-sensing (les HSL) ;

- des composés chimiques capables de stimuler la croissance des populations microbiennes capables de dégrader ces signaux (cette méthode peut être employée en synergie avec l'application d'agents de biocontrôle);

- des plantes sauvages ou transgéniques capables de dégrader les HSL.
Ces stratégies d'antivirulence sont en cours d'expérimentations dans différents laboratoires de recherche et certains résultats intéressants peuvent être commentés.

Une large gamme de molécules est actuellement décrite comme susceptible d'affecter directement la signalisation quorum-sensing. Les plus étudiées sont des furanones halogénées de synthèse ou issues d'une algue, Delisea pulchra. Ces molécules, qui n'ont pas été décrites chez la pomme de terre, ont généralement pour cible la protéine bactérienne impliquée dans la perception du signal. Certaines ont été évaluées in vitro par exemple contre Pectobacterium (Rasmussen et Givskov, 2006). L'origine végétale de la plupart de ces composés suggère qu'ils pourraient participer aux systèmes de défense naturels des plantes; leur présence pourrait être recherchée chez la pomme de terre et intégrée dans des processus d'amélioration et de création variétale.

Par ailleurs, de nombreuses bactéries de la rhizosphère et du sol sont capables de dégrader les HSL. Ces bactéries appartiennent à différents groupes bactériens, les firmicutes (Dong et al., 2000; D'Angelo-Picard et al., 2005), les actinobactéries (Uroz et al., 2003; Park et al., 2006) ou les protéobactéries (Uroz et al., 2003 ; Jafra et al., 2006). Certaines de ces bactéries comme Bacillus et Rhodococcus sont d'efficaces agents de biocontrôle contre Pectobacterium in vitro (Dong et al., 2004 ; Uroz et al., 2003).

Ces bactéries dégradant les HSL représentent de 5 à $10 \%$ des populations bactériennes cultivables des sols (D'AngeloPicard et al., 2005). Une étude récente montre qu'il est possible, grâce à l'application de composés chimiques biodégradables, de stimuler la croissance de ces bactéries dégradant les HSL, comme Rhodococcus et Delftia, dans la rhizosphère de la pomme de terre (Cirou et al., 2007). Ces résultats ont été obtenus lors de culture hors sol : leur effet en champ n'a pas été encore étudié.

Enfin, des gènes bactériens impliqués dans la dégradation des HSL ont été identifiés. Ils appartiennent à trois familles distinctes de protéines : deux familles de lactonase dont les archétypes ont été identifiés chez Bacillus (Dong et al., 2000) et Rhodococcus (Park et al., 2006) et une famille d'amidohydrolase chez Ralstonia (Lin et al., 2003). Le gène bactérien codant une lactonase de Bacillus a été introduit dans la pomme de terre par transgenèse. Il confère aux plantes, y compris le tubercule, la 
propriété de dégradation des HSL. Ces plantes transgéniques sont plus résistantes que leurs parents sauvages à une infection par Pectobacterium (Fray et al., 1999; Dong et al., 2001). L'impact environnemental de plantes exprimant ce type de transgène est en cours d'évaluation.

\section{Conclusions et perspectives}

Les dégâts et pertes économiques causés par les Pectobacteria peuvent être chiffrés en millions d'euros, que ce soit sur le territoire national ou pour d'autres pays de la communauté européenne, encore plus touchés par ces pathogènes, comme les Pays-Bas et l'Allemagne. La recherche et l'application de moyens de lutte efficaces permettant de protéger toutes les étapes de production et de stockage des tubercules représentent donc un véritable enjeu pour les années à venir. Grâce à une meilleure compréhension des mécanismes qui régissent l'apparition et le développement de la maladie, de nouvelles méthodes de lutte chimique, génétique et biologique ont été proposées. Certaines de ces méthodes ont montré leur efficacité au laboratoire et devront être validées au champ. Un espoir conséquent repose en particulier sur des nouvelles méthodes visant à perturber la communication cellulaire des Pectobacteria, que ce soit via l'utilisation de plantes modifiées, d'inocula antagonistes ou de stimulants organiques des bactéries dégradant les signaux quorum-sensing. Ces techniques ne sont pas exclusives et pourraient être associées dans une pratique de lutte intégrée. L'impact environnemental et l'efficacité de ces nouvelles stratégies de lutte doivent être évalués.

\section{Remerciements}

Nous remercions le Comité Nord-Plants de pommes de terre, la région HauteNormandie et le ministère de l'Écologie et du Développement durable pour le soutien financier qu'ils apportent à nos laboratoires dans le contexte de ces nouveaux travaux de recherche.

\section{Références}

Cirou A, Diallo S, Kurt C, et al. Growth promotion of quorum-quenching bacteria in the rhizosphere of Solanum tuberosum. Environ Microbiol 2007 ; 9 : 1511-22.

Compant S, Duffy B, Nowak J, et al. Use of plant growth promoting bacteria for biocontrol of plant diseases: principles, mechanisms of action, and future prospects. Appl Environ Microbiol 2005 ; 71 : 4951-9.

Cronin D, Moënne-Loccoz Y, Fenton A, et al. Ecological interaction of a biocontrol Pseudomonas fluorescens strain producing 2,4 diacetylphloroglucinol with the soft rot potato pathogen Erwinia carotovora subsp. atroseppathogen Erwinia carotovora subsp. atrosep-
tica. FEMS Microbiol Ecol 1997 ; 23 : 95-106.

D'Angelo-Picard C, Faure D, Penotl, et al. Diversity of $N$-acylhomoserine lactoneproducing and-degrading bacteria in soil and tobacco rhizosphere. Environ Microbiol 2005 ; $7: 1796-808$

Dong $\mathrm{YH}$, Wang $\mathrm{LH}, \mathrm{Xu} \mathrm{JL}$, et al. Quenching quorum-sensing-dependent bacterial infection by an $N$-acylhomoserine lactonase. tion by an $N$-acylhom
Nature $2001 ; 411: 813-7$.

Dong $\mathrm{YH}, \mathrm{Xu} \mathrm{JL}$, Li XZ, et al. AiiA, an enzyme that inactivates the acylhomoserine lactone quorum-sensing signal and attenuates the virulence of Erwinia carotovora. Proc Nat Acad Sci USA $2000 ; 97: 3526-31$.

Dong $\mathrm{YH}$, Zhang $\mathrm{XF}_{\mathrm{N}} \mathrm{Xu} \mathrm{JL}$, et al. Insecticidal Bacillus thuringiensis silences Erwinia carotovora virulence by a new form of microbial antagonism, signal interference. Appl Environ Microbiol 2004 ; 70 : 954-60.

Duffy B, Scouten A, Raaijmakers M. Pathogen self-defense: mechanisms to counteract microbial antagonism. Annu Rev Phytopathol $2003 ; 41: 501-38$.

Düring $\mathrm{K}$, Porsch $\mathrm{P}$, Fladung $\mathrm{M}$, et al. Transgenic potato plants resistant to the phytopathogenic bacterium Erwinia carotovora. Plant genic bacterium
$1993 ; 3: 587-98$

Epstein L, Bassein S. Patterns of pesticide use in California and the implications for strategies for reduction of pesticides. Annu Rev Phytopathol $2003 ; 41: 351-75$.

Forbes I. States of uncertainty: governing the empire of biotechnology. N Genet Soc 2006 $25: 69-88$.

Fray RG, Throup JP, Daykin M, et al. Plants genetically modified to produce $N$-acylhomoserine lactones communicate with bacteria. Nat Biotechnol $1999 ; 17$ : 1017-20.

Fuqua WC, Winans SC, Greenberg EP. Quorum sensing in bacteria: the LuxR-Luxl family of cell density-responsive transcriptional regulators. J Bacteriol 1994 ; $176: 269-75$.

Gardan L, Gouy C, Christen R, et al. Elevation of three subspecies of Pectobacterium carotovorum to species level: Pectobacterium atrovorum to species level: Pectobacterium atro-
septicum sp. nov., Pectobacterium betavasculorum sp. nov. and Pectobacterium wasabiae sp. nov. Int J Syst Evol Microbiol 2003 ; 53 381-91.

Gebhardt C, Valkonen JPT. Organization of genes controlling disease resistance in the potato genome. Annu Rev Phytopathol 2001 potato genom
$39: 79-102$.

Hélias V. Pectobacterium spp. et Dikeya spp. de la pomme de terre : nouvelle nomenclature pour Erwinia spp., symptomatologie, épidémiologie et prophylaxie. Cah Agric 2008; 17 349-54.
Heuer H, Small K. Bacterial phyllosphere communities of Solanum tuberosum L. and T4-lysozyme-producing transgenic variants. FEMS Microbiol Ecol 1999 ; 28 : 357-71.

Jafra S, Przysowa J, Czajkowski R, et al. Detection and characterization of bacteria from the potato rhizosphere degrading $\mathrm{N}$-acylhomoserine lactone. Can J Microbiol 2006 ; 52 : 1006-15.

Kamysz W, Krolicka A, Bogucka K, et al. Antibacterial activity of synthetic peptides against plant pathogenic Pectobacterium species. J Pathol 2005; 153 : 313-7.

Kloepper JW. Effect of seed piece inoculation with plant growth-promoting rhizobacteria on populations of Erwinia carotovora on potato roots and in daughter tubers. Phytopathology $1983 ; 73: 217-9$.

Latour X, Delorme S, Mirleau P, et al. Identification of traits implicated in the rhizosphere competence of fluorescent pseudomonads: description of a strategy based on population and model strain studies. Agronomie 2003 23 : 397-405.

Latour X, Lemanceau P. Métabolisme énergétique et carboné des Pseudomonas spp. fluorescents à oxydase positive. Agronomie 1997 . $17: 427-43$.

Lin $\mathrm{YH}, \mathrm{Xu} \mathrm{JL}, \mathrm{Hu} \mathrm{J}$, et al. Acylhomoserine lactone acylase from Ralstonia strain XJ12B represents a novel and potent class of quorum-quenching enzymes. Mol Microbiol $2003 ; 47: 849-60$.

Lottmann J, Heuer H, de Vries J, et al. Establishment of introduced antagonistic bacteria in the rhizosphere of transgenic potatoes and their effect on the bacterial community. FEMS Microbiol Ecol $2000 ; 33: 41-9$.

Lottmann J, Heuer H, Smalla K, et al. Influence of transgenic T4-lysozyme-producing potato plants on potentially beneficial plant associated bacteria. FEMS Microbiol Ecol 1999; 29 : 365-77.

Lyon GD. The biochemical basis of resistance of potatoes to soft rot Erwinia spp.-a review. Plant Pathol 1989 ; 38 : 313-39.

Maë A, Montesano M, Koiv V, et al. Transgenic plants producing the bacterial pheromone $\mathrm{N}$-acylhomoserine lactone exhibit enhanced resistance to the bacterial phytopathogen Erwinia carotovora. Mol Plant Microbe Inte ract $2001 ; 14: 1035-42$.

Moreau M, Feuilloley MGJ, Orange N, et al. Lethal effect of the gliding arc discharges on Erwina spp. J Appl Microbiol 2005; 98 : 1039-46.

Myskja BK. The moral difference between intragenic and transgenic modification of plants. J Agric Environ Ethics 2006; 19 225-38.

Park SY, Hwang BJ, Shin $\mathrm{MH}$, et al. $\mathrm{N}$-acylhomoserine lactonase producing Rhodococcus spp. with different AHL-degrading activities. FEMS Microbiol Lett 2006; 261: 102-8.

Pasco C. Comportement variétal de pomme de terre vis-à-vis des pourritures molles : test sur tubercules en conditions contrôlées. Cah Tech INRA 2005 : 183-7.

Pasco C. Comportement variétal de pomme de terre vis-à-vis des pourritures molles et de la jambe noire : test au champ et en conditions contrôlées. Cah Tech INRA 2005 : 177-81. 
Pérombelon MCM. Potato diseases caused by soft rot erwinias: an overview of pathogenesis. Plant Pathol 2002 ; 51 : 1-12.

Priou S, Jouan B. Les maladies provoquées par les bactéries pathogènes du genre Erwinia. In : Rousselle P, Robert Y, Crosnier JC, nia. In : Rousselle $\mathrm{P}$, Robert $\mathrm{Y}$, Crosnier JC, Paris (France : Inra éditions, 1996.

Rasmussen TB, Givskov M. Quorum-sensing inhibitors: a bargain of effects. Microbiology $2006 ; 152$ : 895-904.

Reading NC, Sperandio V. Quorum-sensing: the many languages of bacteria. FEMS Microbiol Lett 2006 ; 254 : 1-11.

Rhodes DJ, Logan C. A method for selecting fluorescent pseudomonads inhibitory to seed tuber decay. Potato Res 1987 ; 30 : 603-11.
Smadja B, Latour X, Faure D, et al. Involvement of $N$-acylhomoserine lactones throughout the plant infection by Erwinia carotovora subsp. atroseptica (Pectobacteriu$m$ atrosepticum). Mol Plant Microbe Interact $2004 ; 17: 1269-78$.

Smadja B, Latour X, Trigui S, et al. Thermodependence of growth and enzymatic activities implicated in pathogenicity of two Erwinia carotovora subspecies (Pectobacterium spp.). Can J Microbiol $2004 ; 50: 19-27$.

Uroz S, D'Angelo C, Carlier A, et al. Novel bacteria degrading $N$-acylhomoserine lactones and their use as quenchers of quorum-sensing regulated functions of plant pathogenic bacteria. Microbiology 2003 ; 149 : 1981-9.

Varzakas TH, Arvanitoyannis IS, Baltas $\mathrm{H}$. The politics and science behind GMO acceptance. Crit Rev Food Sci Nutr 2007 ; 47 : 335-61.
Waters CM, Bassler BL. Quorum-sensing: cellto-cell communication in bacteria. Annu Rev Cell Dev Biol 2005 ; 21 : 319-46.

Wisniewski JP, Frangne N, Massonneau A, et al. Between myth and reality: genetically modified maize, an example of a sizeable scientific controversy. Biochimie 2002; 84 : 1095-103.

Xu GW, Gross DC. Selection of fluorescent pseudomonads antagonistic to Erwinia caro tovora and suppressive of potato seed piece decay. Phytopathology $1986 ; 76: 414-22$.

Yaganza ES, Rioux D, Simard M, et al. Ultras tructural alterations of Erwinia carotovora subsp. atroseptica caused by treatment with aluminum chloride and sodium metabisulfite. Appl Environ Microbiol $2004 ; 70$ : 6800-8. 